\title{
ФОТОСИНТЕТИЧЕСКИЕ ПИГМЕНТЫ И ФОТОХИМИЧЕСКАЯ АКТИВНОСТЬ ФОТОСИНТЕТИЧЕСКОГО АППАРАТА ЛИСТЬЕВ КУКУРУЗЫ (Zea mays L.) ПОД ВЛИЯНИЕМ ТИАМЕТОКСАМА*
}

\author{
Д.А. ТОДОРЕНКО1, О.В. СЛАТИНСКАЯ 1 , Ј. НАО², Н.Х. СЕЙФУЛЛИНА 1 , \\ Č.N. RADENOVIĆ ${ }^{3}$, Д.Н. МАTOPИН ${ }^{1}$, Г.В. МАКСИМOB'1, 4
}

В последнее десятилетие неоникотиноидные инсектициды активно используются для защиты растений от вредителей. При этом мало изучено их воздействие на сами растения, в частности на состояние фотосинтезирующих пигментов. В настоящей работе впервые показано, что обработка листьев кукурузы (Zea mays L.) инсектицидом тиаметоксамом (TMX) приводит к снижению функциональной активности фотосистемы II и уменьшению энергизации мембран тилакоидов. Кроме того, эффект воздействия тиаметоксама зависит от генотипа кукурузы. Цель работы заключалась в изучении действия пестицида тиаметоксама на фотосинтетические пигменты и фотохимическую активность фотосинтетического аппарата листьев кукурузы двух генотипов. Опыты проводили в 2018-2019 годах. Объектом исследования служили образцы листьев кукурузы инбредной линии zppl 225 и гибрида zp 341 с высокими показателями всхожести, качества зерна и урожайности (Institut za kukuruz «Zemun Polje», г. Белград, Сербия). Семена проращивали до появления корешков (длина не менее 5 мм), после чего высаживали в грунт (смесь вермикулит: чернозем - 1:1) и выращивали в условиях 16-часового светового дня при постоянной температуре $25^{\circ} \mathrm{C}$. При появлении 3-го настоящего листа (длина более 4 см) растения опрыскивали раствором ТМХ в концентрации 0,2 мг/л. В качестве контроля использовали растения, выращенные в аналогичных условиях без опрыскивания ТМХ. Измерения проводили при достижении 5-м настоящим листом размера 12-14 см. Содержание фотосинтетических пигментов (хлорофилла a, b и каротиноидов) определяли спектрофотометрически в 100 \% экстракте ацетона и рассчитывали по формулам Хольма-Веттштейна. Изменение конформации молекул каротиноидов регистрировали методом спектроскопии комбинационного рассеяния (КР). Светоиндуцированные кинетики быстрой флуоресценции (БФ), замедленной флуоресценции (ЗФ) и модулированного отражения при $\lambda=820$ нм (MP) регистрировали одновременно с помощью мультифункционального анализатора растений М-PEA-2 («Hansatech Instruments», Великобритания). Индукционные кривые БФ (ОЈІР-кривые) анализировали с помощью стандартного ЈIР-теста. Установлено, что в фазу 5-го настоящего листа у инбредной линии zppl 225 содержание хлорофиллов в присутствии ТМХ снижалось с 0,74 до 0,61 мг/г: количество хлорофилла а уменьшилось на $17 \%$, хлорофилла b - на $24 \%$. Напротив, в листьях гибрида zp 341 при воздействии TMX изменений в пигментном составе не выявляли. ОЈІР-кривые у контрольных и обработанных ТМХ листьев имели типичный вид кривых с характерными фазами О-J, J-I и I-P, которые отражали процессы последовательного восстановления переносчиков в электрон-транспортной цепи фотосинтеза (ЭТЦ) между двумя фотосистемами. Воздействие пестицида на листья двух генотипов кукурузы проявлялось в снижении функционального состояния фотосистемы II, определенного по параметру флуоресценции (PI $\mathrm{ABS}_{\mathrm{S}}$, который был получен на основании анализа ОJIP-кривых с помощью JIP-теста. Сравнение PI $_{\mathrm{ABS}}$ в контроле и при воздействии ТМХ выявило статистически значимые (р < 0,05) различия: в листьях у образцов zppl 225 и zp 341, обработанных TMX, параметр PI $_{\mathrm{ABS}}$ снижался соответственно на 29 и 24 \%. В листьях кукурузы были обнаружены изменения в быстрой фазе замедленной флуоресценции, связанные с уменышением энергизации тилакоидной мембраны при воздействии ТМХ. Анализ максимальных скоростей окисления и восстановления Р700 (кинетика МР) свидетельствует об уменьшении пула акцепторов на акцепторной стороне ФСІ в листьях zppl 225 при воздействии ТМХ. Обнаружено, что реакционные центры (РЦ) ФСІ zр 341 проявили устойчивость к воздействию ТМХ (отсутствие изменений в редокс-превращениях Р700). ТМХ вызывал изменения конформации молекул каротиноидов, но не менял их содержание в листе. Предложенная комбинация методов быстрой флуоресценции, замедленной флуоресценции, модулированного отражения при $\lambda=820$ нм и спектроскопии комбинационного рассеяния может стать основой для формирования эффективной технологии диагностики ранних дефектов состояния фотосинтетических пигментов при попадании пестицидов в интактное растение.

Ключевые слова: кукуруза, пестициды, тиаметоксам, хлорофилл, каротиноид, флуоресценция, комбинационное рассеяние, Рамановская спектроскопия.

\footnotetext{
Работа выполнена по гранту РФФИ 20-04-00465 А.
} 
В последнее десятилетие нейроактивные инсектициды на основе никотина (имидаклоприд, ацетамиприд, динотефуран, тиаметоксам) широко используются для защиты растений от вредителей. Неоникотиноиды действуют как агонисты ацетилхолина, связываясь с никотиновыми рецепторами ацетилхолина (nAChR) на постсинаптической мембране, что вызывает блокирование синаптической передачи, торможение проведения возбуждения и гибель насекомого (1). Однако нет единого мнения о влиянии неоникотиноидов на состояние самого растения (2). С одной стороны, неоникотиноиды способны улучшать морфологические и физиологическое характеристики некоторых растений даже в стрессовых условиях, что способствует их росту и повышению урожайности (3), с другой - существуют данные о фитотоксичности неоникотиноидов $(4,5)$.

Известно, что производство кукурузы осложняется воздействием различных абиотических и биотических факторов, а также способностью фитофагов повреждать растение на самых ранних стадиях развития. Перспективно исследовать изменения состояния пигментного состава растений кукурузы при действии неоникотиноидов как в лабораторных, так и полевых условиях.

Фотосинтетический аппарат (ФСА) высших растений, состоящий из пигмент-белковых комплексов, включает два типа пигментов - хлорофиллы и каротиноиды. После поглощения квантов света молекулы пигментов антенны переносят энергию на реакционные центры (РЦ) двух фотосистем, в возбужденном состоянии отдающих электроны акцепторам, которые далее восстанавливают НАД ${ }^{+}$до НАДФН, синтезируют АТФ и впоследствии органические вещества. Состояние ФСА - один из чувствительных индикаторов стресса у растений (6). При его изучении эффективны флуоресцентные методы, обладающие высокой чувствительностью и позволяющие выявить нарушения состояния пигментов задолго до появления морфологических изменений $(7,8)$. При исследовании состояния молекул каротиноидов широко используется метод спектроскопии комбинационного рассеяния света (КР), который позволяет контролировать изменения конформации молекулы каротиноидов антенны фотосистемы II (ФСІІ), оценивая вклад валентных колебаний $\mathrm{C}=\mathrm{C}$-связей $\left(\mathrm{I}_{1520} / \mathrm{I}_{1006}\right)$, переходы из плоской конфигурации молекулы в изогнутую $\left(\mathrm{I}_{960} / \mathrm{I}_{1006}\right)$, а также изменения длины полиеновой цепи $\left(\mathrm{I}_{1520} / \mathrm{I}_{1160}\right)(9)$.

В настоящей работе впервые показано, что обработка листьев кукурузы инсектицидом тиаметоксамом (ТМХ) приводит к снижению функциональной активности фотосистемы II и уменышению энергизации мембран тилакоидов. Кроме того, эффект воздействия тиаметоксама зависит от генотипа кукурузы. Так, у инбредной линии zppl 225 показано уменьшение содержания хлорофилла и пула акцепторов на акцепторной стороне ФСІ. Тиаметоксам вызывает противоположные изменения конформации молекул каротиноидов в антенне, но не меняет их содержание.

Цель работы заключалась в изучении действия пестицида тиаметоксама на фотосинтетические пигменты и фотохимическую активность фотосинтетического аппарата листьев кукурузы двух генотипов.

Методика. Опыты проводили в 2018-2019 годах. Исследовали образцы листьев кукурузы (Zea mays L.) инбредной линии zppl 225 и гибрида zp 341 с высокими показателями всхожести, качества зерна и урожайности (Institut za kukuruz «Zemun Polje», г. Белград, Сербия) (10). Семена проращивали до появления корешков (длина не менее 5 мм), после чего выса- 
живали в грунт (смесь вермикулита с черноземом 1:1) и выращивали в условиях 16-часового светового дня при постоянной температуре $25{ }^{\circ} \mathrm{C}$. При появлении 3-го настоящего листа длиной более 4 см (10-е сут после проращивания) растения опрыскивали раствором тиаметоксама [5-метил-3-(2-хлортиазол-5-илметил)-1,3,5-оксадиазинан-4-илиден-N-нитроамин] в концентрации 0,2 мг/л. В качестве контроля использовали листья кукурузы, выращенные в аналогичных условиях без опрыскивания тиаметоксамом. Измерения проводили при достижении 5-м настоящим листом размера $12-14 \mathrm{~cm}$.

Содержание фотосинтетических пигментов (хлорофиллов a, b и каротиноидов) определяли спектрофотометрически в $100 \%$ экстракте ацетона на приборе Hitachi-557 («Hitachi», Япония) и рассчитывали по формулам Хольма-Веттштейна (11). Изменение конформации молекул каротиноидов регистрировали методом спектроскопии КР при помощи КРспектрометра ДФС 24 («ЛОМО», Россия) с лазером 473 нм (Ciel, «Eurolase», Германия) и системой регистрации МОРС 1/3648 («ЛОМО», Россия) на базе линейной ПЗС-матрицы TCD1304DG («Toshiba», Япония) с фильтром LPO2-473RS-50 («Shemrock», США). В эксперименте фрагмент листовой пластинки фиксировали на предметном столике и регистрировали сигнал КР в течение 5 с, мощность лазера на образце 3 мВт.

Светоиндуцированные кинетики быстрой флуоресценции (БФ), замедленной флуоресценции (ЗФ) и модулированного отражения при $\lambda=820$ нм (MP) регистрировали одновременно с помощью мультифункционального анализатора растений М-PEA-2 («Hansatech Instruments», Великобритания). Интенсивность действующего света и продолжительность освещения составляли соответственно 3000 мкмоль квантов $\cdot \mathrm{M}^{-2} \cdot \mathrm{c}^{-1}$ и 60 с. Измерения проводили на адаксиальной стороне интактных листьев, помещенных в измерительный блок в специальной клипсе-прищепке. Перед измерением растения адаптировали к темноте в течение 15 мин, чтобы реакционные центры (РЦ) фотосистем перешли в «открытое» состояние с окисленным $\mathrm{Q}_{\mathrm{A}}$. Три сигнала регистрировали при чередовании световых и темновых интервалов, длительность которых подробно описана R.J. Strasser с соавт. (12).

Индукционные кривые БФ (ОЈР-кривые) анализировали с помощью стандартного JIP-теста, описывающего потоки энергии через различные участки электрон-транспортной цепи фотосинтеза (13) при 20 мкс $\left(\mathrm{F}_{\mathrm{O}}\right), 270$ мкс $\left(\mathrm{F}_{270 \mathrm{мкc}}\right), 2$ мс $\left(\mathrm{F}_{\mathrm{J}}\right), 30$ мс $\left(\mathrm{F}_{\mathrm{I}}\right)$ и максимальной степени флуоресценции $\left(\mathrm{F}_{\mathrm{M}}\right)$. Эти характеристики использовали для расчета максимального квантового выхода фотохимии ФСІІ как отношения переменной флуоресценции $\left(\mathrm{F}_{\mathrm{V}}\right)$ к максимальной $\left(\mathrm{F}_{\mathrm{M}}\right) \mathrm{F}_{\mathrm{V}} / \mathrm{F}_{\mathrm{M}}=1-\mathrm{F}_{\mathrm{O}} / \mathrm{F}_{\mathrm{M}}$, вероятности транспорта электронов за пределы $\mathrm{Q}_{\mathrm{A}}^{-} \psi_{\text {Ео }}=1-\left(\mathrm{F}_{\mathrm{J}}-\mathrm{F}_{\mathrm{O}}\right) /\left(\mathrm{F}_{\mathrm{M}}-\mathrm{F}_{\mathrm{O}}\right)$, потока энергии (ABS), поглощаемой одним активным реакционным центром $(\mathrm{RC})$, как $\mathrm{ABS} / \mathrm{RC}=4 \times\left(\mathrm{F}_{270 \mathrm{мкс}}-\mathrm{F}_{\mathrm{O}}\right) \times\left(\mathrm{F}_{\mathrm{M}}-\mathrm{F}_{\mathrm{O}}\right)^{-3} \times\left(\mathrm{F}_{\mathrm{J}}-\mathrm{F}_{\mathrm{O}}\right)^{-1} \times \mathrm{F}_{\mathrm{M}}$ и показателя функциональной активности ФСII, отнесенной к поглощаемой энергии (индекс производительности ФСII): $\mathrm{PI}_{\mathrm{ABS}}=(\mathrm{ABS} / \mathrm{RC})^{-1} \times \mathrm{F}_{\mathrm{V}} / \mathrm{F}_{\mathrm{M}} \times$ $\times\left(1-\mathrm{F}_{\mathrm{V}} / \mathrm{F}_{\mathrm{M}}\right)^{-1} \times \psi_{\text {Еo }} \times\left(1-\psi_{\text {Eo }}\right)^{-1}(13)$.

Кинетики модулированного отражения/поглощения света при $\lambda=820$ нм (МР) характеризуют окислительно-восстановительные превращения пигмента ФСІ (Р700) и пластоцианина (12). В нашем исследовании сигнал МР был нормирован по $\mathrm{MP}_{0}$ и представлен как $\mathrm{MP} / \mathrm{MP}_{0}-1$, где $\mathrm{MP}_{0}-$ интенсивность сигнала рассеяния при 0,7 мс. 
Статистическую обработку полученных данных проводили, используя непараметрический критерий для множества независимых переменных Краскела-Уоллиса (Kruskal-Wallis test) с помощью пакета Statistica v.10 («StatSoft, Inc.», США). Значения p $<0,05$ считались статистически значимыми. В таблице представлены значения среднего $(M)$ и стандартные ошибки среднего ( \pm SEM).

Результаты. Пигментный состав листьев мало различался у двух генотипов кукурузы (zppl 225 и zp 341), но после действия тиаметоксама были обнаружены явные изменения, зависящие от генотипа. Так, в листьях у инбредной линии zppl 225 в присутствии ТМХ выявили снижение содержания хлорофиллов с 0,74 до 0,61 мг/г: количество хлорофилла а уменьшилось на $17 \%$, хлорофилла $\mathrm{b}-$ на $24 \%$ (p < 0,05). Напротив, при воздействии ТMX на листья гибрида zp 341 изменений в пигментном составе не обнаружили.

Индукционные кривые БФ (ОЈIP) как у контрольных, так и у обработанных ТМХ листьев имели типичный вид кривых с характерными фазами O-J, J-I и I-P, которые отражали процессы последовательного восстановления переносчиков в электрон-транспортной цепи фотосинтеза (ЭТЦ) между двумя фотосистемами (13) (рис. 1, А, Г). Исходный уровень O (origin) соответствует интенсивности флуоресценции при «открытых» РЦ ФСII $\left(\mathrm{F}_{\mathrm{O}}\right)$, когда все акцепторы в фотосистеме II (ФCII) находятся в окисленном состоянии и способны принять электрон. Нарастание флуоресценции в фазе О-Ј обусловлено светоиндуцированным восстановлением состояний $\mathrm{Q}_{\mathrm{A}}^{-}$. Последующие фазы J-I-P отражают дальнейший рост флуоресценции, связанный с накоплением состояний $\mathrm{Q}_{\mathrm{A}}^{-}$в результате восстановления акцепторов $\mathrm{Q}_{\mathrm{B}}$, пула хинонов и первичных акцепторов фотосистемы I (ФСI) (14).
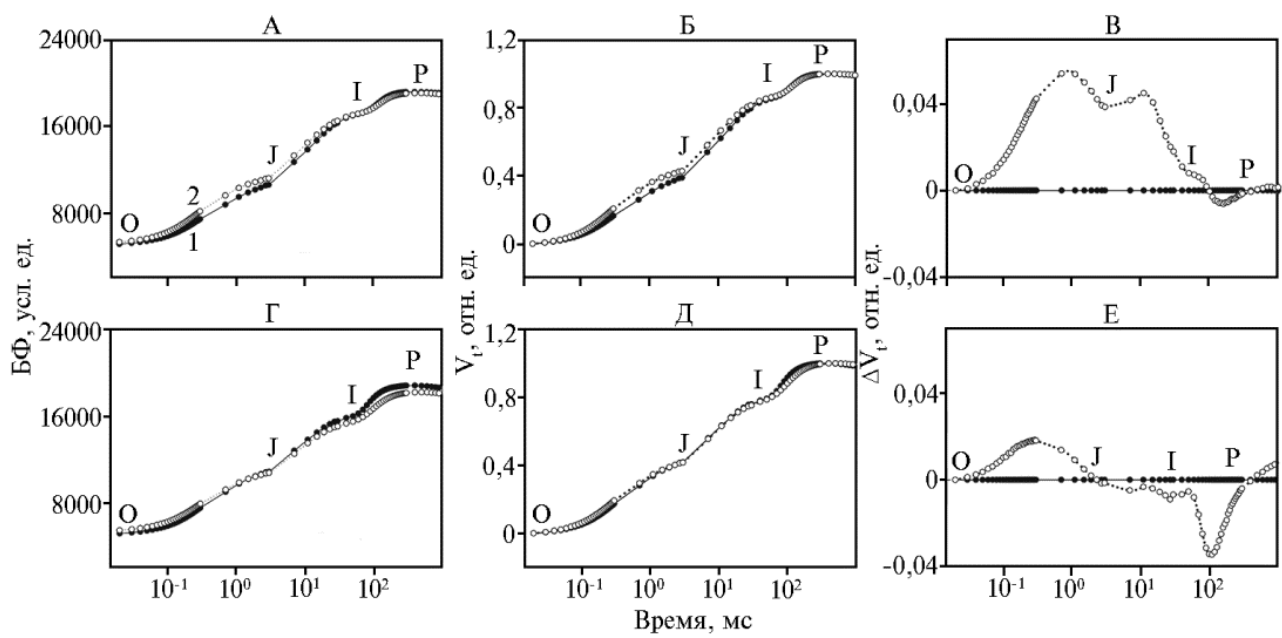

Рис. 1. Индукционные кривые (ОЈIР) быстрой флуоресценции (БФ) листьев кукурузы (Zea mays L.) (A, Г), кривые в виде относительной переменной флуоресценции $V_{t}=\left(F_{t}-F_{O}\right) /\left(F_{M}-F_{O}\right)$

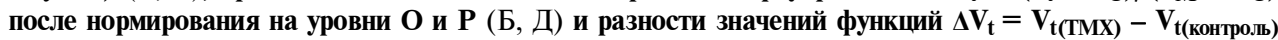
(В, Е) для инбредной линии zppl 225 (А, Б, В) и гибрида zp 341 (Г, Д, Е) в контроле (1) и при опрыскивании тиаметоксамом (2). O, J, I и P - пики на кривой индукции, наблюдаемые соответственно при 20 мкс, 2 мс, 30 мс и 300 мс.

При воздействии ТМХ у инбредной линии zppl 225 и гибрида zp 341 наблюдались небольшие изменения в форме ОJIP (см. рис. 1, А, Г). Для более детального анализа индукционные кривые были дважды нормированы (на уровни О и Р) и представлены в виде относительной перемен- 
ной флуоресценции $\left(\mathrm{V}_{\mathrm{t}}\right)$ (см. рис. 1, Б, Д) и как разность значений функций $\mathrm{V}_{\mathrm{t}}$ между контролем и образцами, обработанными $\mathrm{TMX}\left(\Delta \mathrm{V}_{\mathrm{t}}\right)(\mathrm{cm}$. рис. 1, В, Е). Воздействие пестицида на листья двух генотипов кукурузы проявлялось в увеличении $\Delta \mathrm{V}_{\mathrm{t}}$ в фазе О-J, что свидетельствует о накоплении восстановленного $\mathrm{Q}_{\mathrm{A}}$ в результате увеличения доли $\mathrm{Q}_{\mathrm{B}}$-невосстанавливающих центров ФСІІ, то есть центров, не способных к передаче электрона по электрон-транспортной цепи (ЭТЦ) (15). В листьях инбредной линии zppl 225 изменения в фазе О-Ј оказались более выражены, чем у гибрида zp 341. Кроме того, в листьях zppl 225 эффект воздействия ТМХ сопровождался увеличением амплитуды фазы J-I: $\Delta \mathrm{V}_{\mathrm{t}}$ увеличивалось в фазах O-J и J-I, что свидетельствует о накоплении восстановленных форм $\mathrm{Q}_{\mathrm{A}}$ и молекул пластохинонов, которые не способны к передаче электронов для темновых реакций фотосинтеза (8).

Параметры ЈIР-теста, полученные после анализа индукционных кривых ОЈІР, для листьев растений кукурузы (Zea mays L.) двух генотипов при действии тиаметоксама (TMX) $(M \pm \mathrm{SEM})$

\begin{tabular}{|c|c|c|c|c|}
\hline $\begin{array}{l}\text { Параметр } \\
\text { флуоресценции }\end{array}$ & zppl 225 & zppl $225+$ TMX & zp 341 & zp $341+$ TMX \\
\hline $\mathrm{F}_{\mathrm{V}} / \mathrm{F}_{\mathrm{M}}$ & $0,740 \pm 0,004(100 \%)$ & $0,740 \pm 0,004(99 \%)$ & $0,740 \pm 0,010(100 \%)$ & $0,710 \pm 0,010(97 \%)$ \\
\hline$\psi_{\text {EO }}$ & & & & \\
\hline & & & & \\
\hline BBS & $2,50 \pm 0,10(100 \%)$ & $\%)^{*}$ & & $\%)^{*}$ \\
\hline \multicolumn{5}{|c|}{ 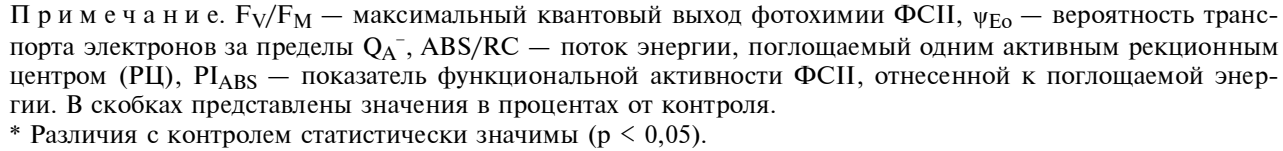 } \\
\hline
\end{tabular}

Индукционные кривые БФ анализировали с помощью JIP-теста (13) (табл.). Известно, что наиболее адекватный параметр JIP-теста - величина отношения $\mathrm{F}_{\mathrm{V}} / \mathrm{F}_{\mathrm{M}}$, которое коррелирует с максимальным квантовым выходом первичной фотохимической реакции ФСІІ и используется как показатель эффективности фотосинтеза (6). Величина $\mathrm{F}_{\mathrm{V}} / \mathrm{F}_{\mathrm{M}}$ в контроле в фазу 5-го листа у zppl 225 и zр 341 составила $0,74 \pm 0,01$. Полученные значения фотосинтетической активности, определенные по $\mathrm{F}_{\mathrm{V}} / \mathrm{F}_{\mathrm{M}}$, были сопоставимы со значениями $\mathrm{F}_{\mathrm{V}} / \mathrm{F}_{\mathrm{M}}$, полученными для растений $\mathrm{C}_{4}$ фотосинтеза (16). При воздействии ТМХ в листьях zppl 225 и zp 341 статистически значимых различий по параметру $\mathrm{F}_{\mathrm{V}} / \mathrm{F}_{\mathrm{M}}$ не выявляли $(\mathrm{p}>0,05)$.

$\mathrm{B}$ отличие от параметра $\mathrm{F}_{\mathrm{V}} / \mathrm{F}_{\mathrm{M}}$, индекс производительности $\mathrm{PI}_{\mathrm{ABS}}$ существенно менялся при воздействии TMХ. Известно, что $\mathrm{PI}_{\mathrm{ABS}}$ коррелирует с жизнеспособностью растения и отражает текущее состояние работы ФСА в условиях стресса (17). Сравнение $\mathrm{PI}_{\mathrm{ABS}}$ в контроле и при воздействии ТМХ выявило статистически значимые $(\mathrm{p}<0,05)$ различия: в листьях у образцов zppl 225 и zр 341, обработанных TMX, параметр PI $_{\mathrm{ABS}}$ снижался соответственно на 29 и $24 \%$.

$\mathrm{PI}_{\mathrm{ABS}}$ - интегральный показатель, который включает в себя три независимых параметра: долю активных РЦ (ABS/RC), эффективность переноса электронов захваченным экситоном в цепи переноса электронов за пределы $\mathrm{Q}_{\mathrm{A}}\left(\psi_{\text {Ео }}\right)$ и вероятность того, что экситон будет связан в РЦ $\left(\mathrm{F}_{\mathrm{V}} / \mathrm{F}_{\mathrm{M}}\right)$ (13). Снижение $\mathrm{PI}_{\mathrm{ABS}}$ в листьях zppl 225 в присутствии ТМХ было обусловлено значительным уменьшением эффективности электронного транспорта на акцепторной стороне ФСII ( $\left.\psi_{\text {Ео }}\right)$, что подтверждается изменениями в индукционных кривых БФ, представленных выше (p < 0,05). Напротив, снижение $\mathrm{PI}_{\mathrm{ABS}}$ в листьях zp 341 могло быть связано с незначительным уменьшением доли активных РЦ (увеличение ABS/RC) и фото- 


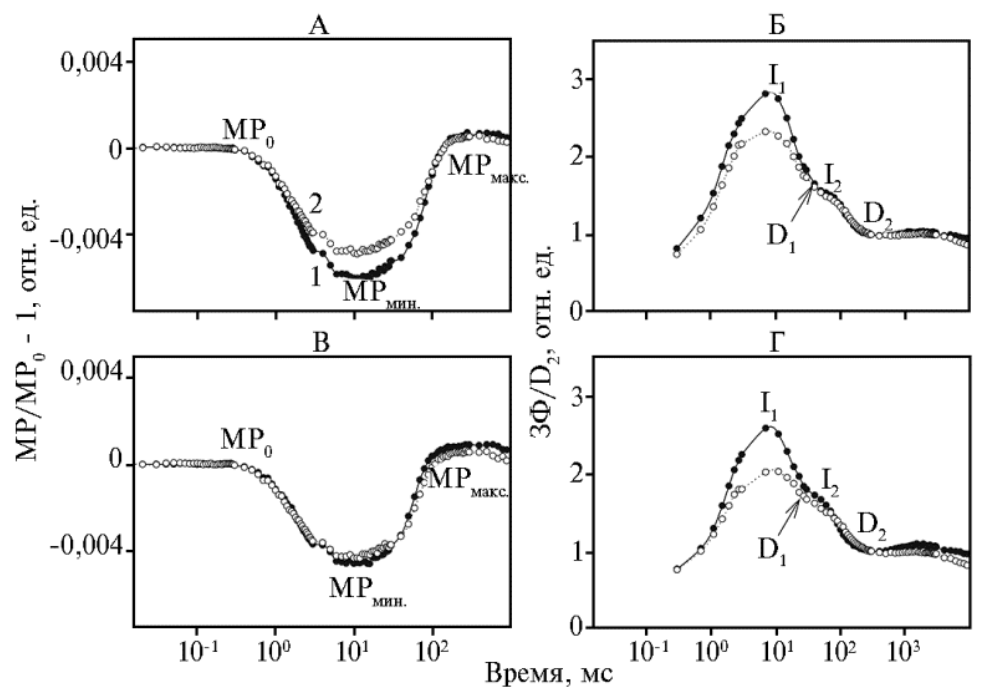

Рис. 2. Светоиндуцированные кинетики модулированного отражения при $\lambda=820$ нм (МР) (A, В) и замедленной флуоресценции (ЗФ), нормированные на минимальный уровень сигнала $D_{2}$ при 200 мс (Б, Г), у листьев кукурузы (Zea mays L.) инбредной линии zppl 225 (А, Б) и гибрида zp $341(\mathrm{~B}$, Г) в контроле (1) и при опрыскивании тиаметоксамом (2). Кинетики МР нормированы и представлены в виде $\mathrm{MP} / \mathrm{MP}_{0}-1$, где $\mathrm{MP}_{0}-$ интенсивность сигнала при 0,7 мс. $\mathrm{I}_{1}, \mathrm{I}_{2}, \mathrm{D}_{1}$, $\mathrm{D}_{2}$ - максимумы и минимумы на индукционной кривой ЗФ.

Окислительно-восстановительные превращения реакционных центров молекул ФСІ (Р700) в присутствии ТМХ мы оценивали по поглощению света при $\lambda=820$ нм (МР) (18) (рис. 2, А, В). Известно, что кинетика светоиндуцированного спада сигнала МР в первые 15-20 мс отражает процесс окисления Р700 (быстрая фаза) и достигает минимума при 20 мс $\left(\mathrm{MP}_{\text {мин }}\right) . \mathrm{MP}_{\text {мин }}$ - переходное устойчивое состояние с равными скоростями окисления и ре-восстановления Р700. Впоследствии скорость ревосстановления преобладает над скоростью окисления за счет донирования электронов от ФСІІ, приводя к уменьшению величины поглощения при $\lambda=820$ нм и росту сигнала МР (медленная фаза) до максимума при $\sim 200$ мс $\left(\mathrm{MP}_{\text {макс }}\right)(12)$.

В присутствии ТМХ в листьях кукурузы инбредной линии zppl 225 и гибрида zр 341 были выявлены изменения в окислительно-восстановительных превращениях Р700 (см. рис. 2, А, В). Отметим, что у хлорофилла в листьях zppl 225 были обнаружены изменения в быстрой и медленной фазах МР, связанные с уменьшением амплитуды при 20 мс (см. рис. 2, А). Анализ максимальных скоростей окисления и восстановления Р700 (кинетика МР) свидетельствует об уменьшении образования окисленных и увеличении - восстановленных форм акцепторов ФСІ (Р700) при воздействии ТМХ на листья zppl 225. Вероятно, это связано с уменышением пула акцепторов, таких как P700, на акцепторной стороне ФСІ. Напротив, РЦ ФCI листьев гибрида zp 341 проявляли резистентность к воздействию TMХ (отсутствие изменений в МР кинетиках) (см. рис. 2, В).

Степень энергизации тилакоидных мембран мы оценивали по пикам $\mathrm{I}_{1}$ и $\mathrm{I}_{2}$ замедленной флуоресценции, измеренной в микросекундном временно́м диапазоне (0-0,09 мс). Известно, что ЗФ пропорциональна скорости реакций рекомбинаций в ФСІІ, на которые влияет энергизация тилакоидной мембраны (6). В процессе работы мы анализировали быст- 
рую фазу ЗФ, связанную с формированием потенциала на мембране. Известно, что быстрая фаза ЗФ имеет два пика $\mathrm{I}_{1}(\sim 7 \mathrm{Mc})$ и $\mathrm{I}_{2}(\sim 100 \mathrm{Mc})$, после чего падает до минимума $\mathrm{D}_{2}$ ( 200 мс) (19). На рисунке 2 (Б, Г) представлены индукционные кривые ЗФ листьев zppl 225 и zр 341, обработанных ТМХ, нормированные на уровень минимальной флуоресценции $\mathrm{D}_{2}$. При действии TMX в листьях zppl 225 и zp 341 снижалась амплитуда пика $\mathrm{I}_{1}$ по сравнению с контролем соответственно на 23 и $21 \%$. Уменьшение пика $\mathrm{I}_{1}$ в микросекундном временно́м диапазоне могло быть вызвано снижением скорости ре-окисления $\mathrm{Q}_{\mathrm{A}}$ (нарушение на акцепторной стороне ФСІІ) и/или уменьшением восстановления $\mathrm{Z}^{+}$от $4 \mathrm{MnCa}$ кластера кислород-выделяющего комплекса (КВК) (19).

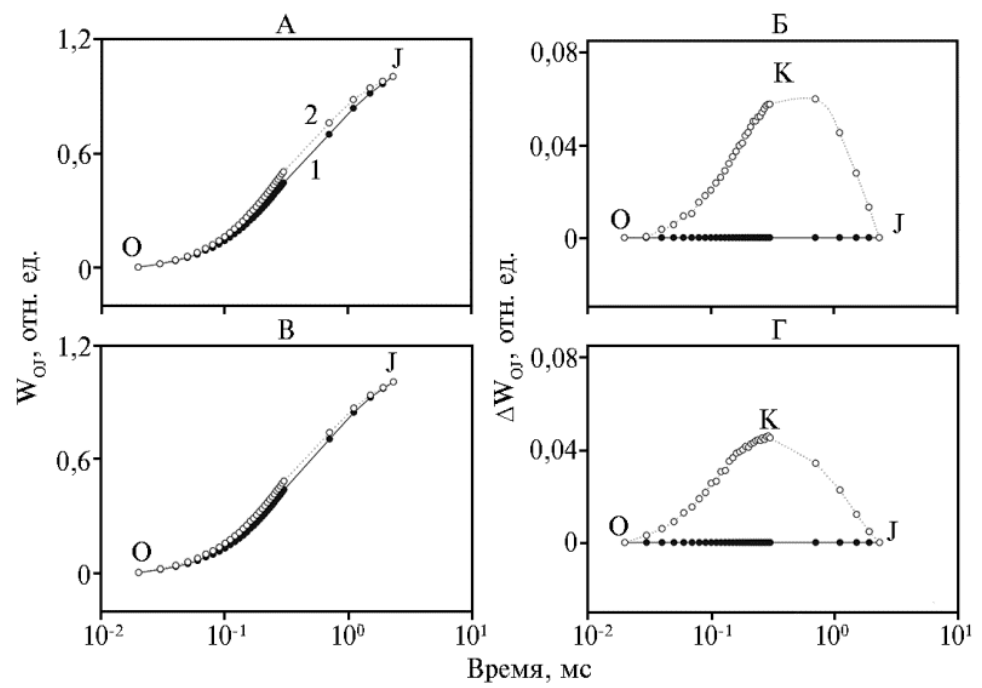

Рис. 3. Относительная переменная флуоресценция $\mathrm{W}_{\mathrm{OJ}}=\left(\mathrm{F}_{\mathrm{t}}-\mathrm{F}_{\mathrm{O}}\right) /\left(\mathrm{F}_{\mathrm{J}}-\mathrm{F}_{\mathrm{O}}\right)$ между уровнями $\mathrm{O}$

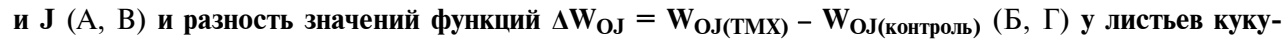
рузы (Zea mays L.) инбредной линии zppl 225(A, Б) и гибрида zp 341 (В, Г) в контроле (1) и при опрыскивании тиаметоксамом (2). О, K, J - пики на кривой индукции, наблюдаемые соответственно при 20 мкс, 300 мкс и 2 мс.

Состояние КВК и его способность донировать электрон для $\mathrm{P} 680^{+}$ через $\mathrm{Z}^{+}$мы контролировали по появлению дополнительного пика на индукционной кривой БФ. Для этого рассчитывали относительную флуоресценцию между пиками $\mathrm{O}$ и $\mathrm{J}$ как $\mathrm{W}_{\mathrm{OJ}}=\left(\mathrm{F}_{\mathrm{t}}-\mathrm{F}_{\mathrm{O}}\right) /\left(\mathrm{F}_{\mathrm{J}}-\mathrm{F}_{\mathrm{O}}\right)$ (см. рис. 3, A, В) и разность значений функций между контролем и образцами, обработанными ТМХ как $\Delta \mathrm{W}_{\mathrm{OJ}}=\mathrm{W}_{\mathrm{OJ}(\mathrm{TMX})}-\mathrm{W}_{\mathrm{OJ}(\text { контроль) }}$ (см. рис. 3, Б, Г). В листьях zppl 225 и zp 341 при действии ТМХ был обнаружен дополнительный пик между О и J при 300 мкс (пик К) (см. рис. 3, Б, Г), который характерен для высокотемпературного стресса за счет ограничения переноса электронов к РЦ из-за нарушений в работе КВК (13).

В спектрах комбинационного рассеивания (КР) листьев кукурузы были выявлены характерные для каротиноидов полосы $(960,1006,1156$, 1190, 1200 и $1520 \mathrm{~cm}^{-1}$ ), обусловленные валентными колебаниями электронов в полиеновой цепочке молекулы (рис. 4). Максимальные изменения амплитуды полос спектра КР наблюдались для соотношений $\mathrm{I}_{1520} / \mathrm{I}_{1006}$ (вклад валентных колебаний $-\mathrm{C}=\mathrm{C}-$ связей полиеновой цепи каротиноида), $\mathrm{I}_{1520} / \mathrm{I}_{1160}$ (изменение вклада $-\mathrm{C}=\mathrm{C}-$ связей по отношению $\mathrm{K}-\mathrm{C}-\mathrm{C}-$ связям полиеновой цепи) и $\mathrm{I}_{1006} / \mathrm{I}_{960}$ (расположение полиеновой цепи каротиноида относительно пиррольных колец) (20). Наличие в спектрах КР 
пиков на 1190 и $1200 \mathrm{~cm}^{-1}$ указывало на состояние каротиноидов, характерное для 15-сіs конформации (данные не представлены).

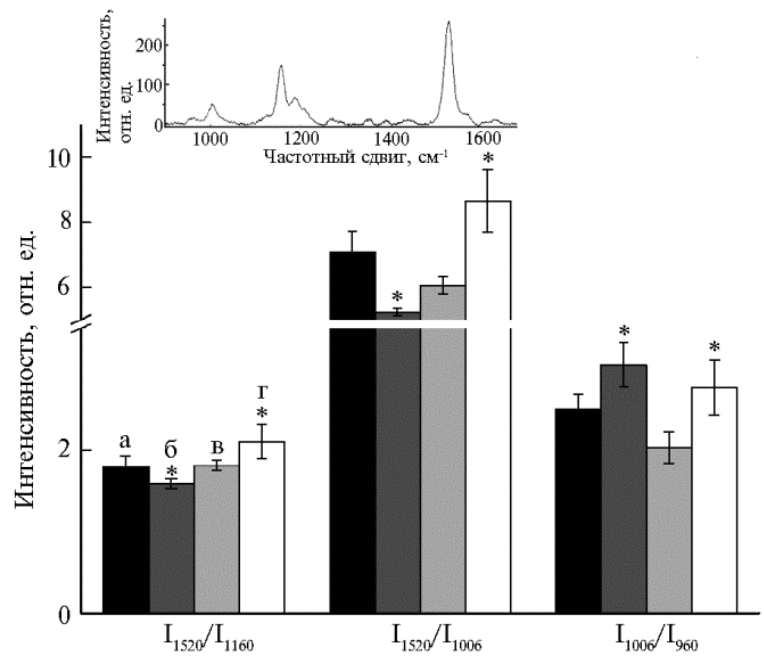

Рис. 4. Изменение спектров комбинационного рассеивания (KP) каротиноидов в листьях кукурузы (Zea mays L.) двух генотипов при опрыскивании тиаметоксамом (TMX): a - инбредная линия zppl 225, б - zppl $225+$ TMX, в - гибрид zp 341, г - гибрид zp $341+$ ТМХ. Звездочкой обозначены статистически значимые различия $(\mathrm{p}<0,05)$. На вставке представлен спектр КР каротиноида листа кукурузы инбредной линии zppl 225.

ноидов, но не их содержания.

В настоящей работе мы исследовали влияние пестицида ТМХ на фотосинтетические пигменты и фотохимическую активность фотосинтетического аппарата листьев кукурузы двух генотипов с помощью современных спектральных и флуоресцентных методов.

Инбредная линия кукурузы zppl 225 была подвержена большим изменениям при опрыскивании листьев ТMX, чем гибрид zp 341. У инбредной линии zppl 225 выявлено уменьшение содержания хлорофиллов, которое, вероятно, свидетельствует о том, что при действии ТМХ снижается синтез этих пигментов. Отметим, что в листьях zppl 225 выявлено уменьшение соотношения хлорофиллы $(\mathrm{a}+\mathrm{b}) /$ каротиноиды, что, как правило, коррелирует с увеличением содержания каротиноидов в условиях стресса (21). Напротив, у листьев гибрида zр 341 изменений в пигментном составе не выявлено. Ранее на примере хлопка показано, что неоникотиноиды не влияют на содержание хлорофиллов, тогда как у листьев бамии при воздействии ацетамиприда зарегистрировано постепенное увеличение общего содержания хлорофилла (22). В то же время ТМХ может выступать в качестве биоактиватора, увеличивая физиологическую и метаболическую активность такой культуры, как рис (23)

Влияние ТМХ на состояние ФСІІ, ФСІ, электронных переносчиков между фотосистемами в листьях кукурузы zppl 225 и zp 341 оценивали, исходя из набора параметров, полученных после анализа кинетических кривых БФ, ЗФ и МР. Анализ кинетических кривых БФ с помощью JІР-теста, который описывает потоки энергии через различные участки электрон-транспортной цепи фотосинтеза (13), позволил выявить, что TMX оказывал незначительное (p > 0,05) воздействие на фотохимию 
ФCII $\left(\mathrm{F}_{\mathrm{V}} / \mathrm{F}_{\mathrm{M}}\right)$ в листьях двух изученных генотипов. Обнаружены изменения на акцепторной стороне ФСІІ, связанные со снижением вероятности электронного транспорта после акцептора $\left.\mathrm{Q}_{\mathrm{A}}^{-}{ }^{-} \psi_{\text {Ео }}\right)$, в листьях инбредной линии zppl 225. Снижение эффективности электронного транспорта на акцепторной стороне ФСІІ ( $\psi_{\text {Ео }}$ ) вызывало уменьшение функциональной активности ФСII (PI $\left.{ }_{\mathrm{ABS}}\right)$. Другие неоникотинодные пестициды, такие как ацетамиприд, также не оказывают воздействия на $\mathrm{F}_{\mathrm{V}} / \mathrm{F}_{\mathrm{M}}$ и приводят $\mathrm{K}$ значительному снижению функциональной активности ФСII за счет уменьшения размера пула и снижения электронного транспорта на акцепторной стороне ФСІІ у цианобактерий Synechocystis sp. (24).

Таким образом, при опрыскивании растений кукурузы пестицидом тиаметоксамом (ТМХ) было выявлено снижение содержания хлорофилла в листьях инбредной линии zppl 225 по сравнению с гибридом zp 341. Кроме того, в листьях zppl 225 обнаружено уменьшение пула акцепторов на акцепторной стороне фотосистемы I (ФСI). Установлено, что ТМХ воздействует на функциональную активность фотосистемы II (ФСII), уменьшая параметр $\mathrm{PI}_{\mathrm{ABS}}$, и снижает формирование потенциала на тилакоидной мембране $\left(\mathrm{I}_{1}\right)$ как у zppl 225, так и у zp 341. ТMХ вызывал противоположные изменения конформации молекул каротиноидов в антенне, но не менял их содержание, что, по-видимому, связано с более эффективным рассеянием избыточной энергии через систему каротиноидов в листьях zp 341 по сравнению c zppl 225. Предложенная комбинация методов позволяет эффективно оценивать функциональное состояние фотосинтетического аппарата в листьях в полевых и лабораторных условиях как при биомониторинге, так и при сравнении различных линий сельскохозяйственных культур.

\section{ЛИТЕРАТУРА}

1. Ensley S.M. Neonicotinoids. Veterinary toxicology (third edition). Basic and clinical principles /R.C. Gupta (ed.). Academic Press, 2018: 521-524 (doi: 10.1016/C2016-0-01687-X).

2. Georgieva M., Tsenov B., Dimitrova A. Dual effects of N-nitroguanidine neonicotinoids on plants. Genetics and Plant Physiology, 2017, 7(3-4): 107-120.

3. da Silva Almeida A., Villela F.A., Nunes J.C., Meneghello G.E., Jauer A. Thiamethoxam: an inseticide that improve seed rice germination at low temperature insecticides. In: Insecticides Development of safer and more effective technologies /T. Trdan (ed.). IntechOpen, 2013: 417-426 (doi: 10.5772/53207).

4. Shakir S.K., Irfan S., Akhtar B., Rehman S.U., Daud M.K., Taimur N., Azizullah A. Pesticideinduced oxidative stress and antioxidant responses in tomato (Solanum lycopersicum) seedlings. Ecotoxicology, 2018, 27(7): 919-935 (doi: 10.1007/s10646-018-1916-6).

5. Çavuşoğlu K., Yalçın E., Türkmen Z., Yapar K., Sağır S. Physiological, anatomical, biochemical, and cytogenetic effects of thiamethoxam treatment on Allium cepa (amaryllidaceae) L. Environmental Toxicology, 2012, 27(11): 623-670 (doi: 10.1002/tox.20680).

6. Маторин Д.Н., Рубин А.Б. Флуоресиенция хлорофилла высших растений и водорослей. М.Ижевск, 2012.

7. Matorin D.N., Todorenko D.A., Seifullina N.Kh, Zayadan B.K., Rubin A.B. Effect of silver nanoparticles on the parameters of chlorophyll fluorescence and $\mathrm{P}_{700}$ reaction in the green alga Chlamydomonas reinhardtii. Microbiology, 2013, 82(6): 809-814 (doi: 10.1134/S002626171401010X).

8. Kalaji H.M., Jajoo A., Oukarroum A., Brestic M., Zivcak M., Samborska I.A., Cetner M.D., Łukasik I., Goltsev V., Ladle R.J., Dąbrowski P., Ahmad P. The use of chlorophyll fluorescence kinetics analysis to study the performance of photosynthetic machinery in plants. In: Emerging Technologies and Management of Crop Stress Tolerance. V. 2 /P. Ahmad, S. Rasool (eds.). Elsevier, USA, 2014: 347-384 (doi: 10.1016/B978-0-12-800875-1.00015-6).

9. Merlin J.C. Resonance Raman spectroscopy of carotenoids and carotenoid-containing systems. Pure and Applied Chemistry, 1985, 57(5): 785-792 (doi: 10.1351/pac198557050785).

10. Тютяев Е.В., Шутова В.В., Максимов Г.В., Раденович Ч.Н., Гродзинский Д.М. Состояние фотосинтетических пигментов в листьях инбредных линий и гибридов кукурузы. 
Физиология растений и генетика, 2015, 47(2): 147-159.

11. von Wettstein D. Chlorophyll-letale und der submikroskopische Formwechsel der Plastiden. Experimental Cell Research, 1957, 12(3): 427-506 (doi: 10.1016/0014-4827(57)90165-9).

12. Strasser R.J., Tsimilli-Michael M., Qiang S., Goltsev V. Simultaneous in vivo recording of prompt and delayed fluorescence and $820-\mathrm{nm}$ reflection changes during drying and after rehydration of the resurrection plant Haberlea rhodopensis. Biochimica et Biophysica Acta (BBA) - Bioenergetics, 2010, 1797(6-7): 1313-1326 (doi: 10.1016/j.bbabio.2010.03.008).

13. Strasser R.J., Tsimilli-Michael M., Strivastava A. Analysis of the chlorophyll a fluorescence transient. In: Chlorophyll a fluorescence: advances in photosynthesis and respiration series, V. 19 /G.C. Papageorgiou, Govindjee (eds.). Springer, Dordrecht, 2004: 321-362 (doi: 10.1007/9781-4020-3218-9_12).

14. Stirbet A., Govindjee. Chlorophyll a fluorescence induction: a personal perspective of the thermal phase, the J-I-P rise. Photosynthesis Research, 2015, 113(1-3): 15-61 (doi: 10.1007/s11120012-9754-5).

15. Lazár D. The polyphasic chlorophyll a fluorescence rise measured under high intensity of exciting light. Functional Plant Biology, 2006, 33(1): 9-30 (doi: 10.1071/FP05095).

16. Nieva F.J.J., Castellanos E.M., Figueroa M.E., Gil F. Gas exchange and chlorophyll fluorescence of C3 and C4 saltmarsh species. Photosynthetica, 1999, 36(3): 397-406 (doi: 10.1023/A:1007024019133).

17. Živčák M., Olšovská K., Slamka P., Galambošová J., Rataj V., Shao H.B., Brestič M. Application of chlorophyll fluorescence performance indices to assess the wheat photosynthetic functions influenced by nitrogen deficiency. Plant, Soil and Environment, 2014, 60(5): 210-215 (doi: 10.17221/73/2014-PSE).

18. Schansker G., Srivastava A., Govindjee, Strasser R.J. Characterization of the 820-nm transmission signal paralleling the chlorophyll a fluorescence rise (OJIP) in pea leaves. Functional Plant Biology, 2003, 30(7): 785-796 (doi: 10.1071/FP03032).

19. Goltsev V., Chernev P., Zaharieva I., Strasser R.J. Delayed fluorescence in photosynthesis. Photosynthesis Research, 2009, 101(2-3): 217-232 (doi: 10.1007/s11120-009-9451-1).

20. Кэри П. Применение спектроскопии КР и РКР в биохимии /Под ред. Б.В. Локшина. М., 1985.

21. Соловченко А.Е., Мерзляк М.Н. Оптическое экранирование как фотозащитный механизм растений. М., 2010.

22. Preetha G., Stanley J. Influence of neonicotinoid insecticides on the plant growth attributes of cotton and okra. Journal of Plant Nutrition, 2012, 35(8): 1234-1245 (doi: 10.1080/01904167.2012.676134).

23. Macedo W.R., Araújo D.K., de Camargo e Castro P.R. Unravelling the physiologic and metabolic action of thiamethoxam on rice plants. Pesticide Biochemistry and Physiology, 2013, 107(2): 224-249 (doi: 10.1016/j.pestbp.2013.08.001).

24. Li L., Chen Xi., Zhang D., Pan X. Effects of insecticide acetamiprid on photosystem II (PSII) activity of Synechocystis sp. (FACHB-898). Pesticide Biochemistry and Physiology, 2010, 98(2): 300-304 (doi: 10.1016/j.pestbp.2010.06.022).

ІФГБОУ ВО Московский государственный университет им. М.В. Ломоносова, Биологический факультет, 119234 Россия, г. Москва, Ленинские горы, МГУ, 1, стр. 12, Биологиче-

Поступила в редакцию ский факультет МГУ,

e-mail: dariatodor@mail.ru $₫$, slatolya@mail.ru, matorin@biophys.msu.ru, maksimov@mail.ru;

2Shenzhen MSU-BIT University,

No 299, Ruyi Road, Longgang District,

Shenzhen, Guangdong, 518172 China,

e-mail: haojr@szmsubit.edu.cn;

3 Institut za kukuruz «Zemun Polje»,

11185 Beograd-Zemun, ul. Slobodana Bajica 1, Srbija,

e-mail: radenovic@sbb.rs;

${ }^{4}$ ФГАОУ ВО Национальный исследовательский

технологический университет МИСиС,

119049 Россия, г. Москва, Ленинский просп., 4,

e-mail: gmaksimov@mail.ru

Sel’skokhozyaistvennaya biologiya [Agricultural Biology], 2020, V. 55, № 1, pp. 66-76

\title{
PHOTOSYNTHETIC PIGMENTS AND PHYTOCHEMICAL ACTIVITY OF PHOTOSYNTHETIC APPARATUS OF MAIZE (Zea mays L.) LEAVES UN- DER THE EFFECT OF THIAMETHOXAM
}

\author{
D.A. Todorenko ${ }^{1}$, O.V. Slatinskaya ${ }^{1}$, J. Hao ${ }^{2}$, N.Kh. Seifullina ${ }^{1}$, Č.N. Radenović3,
}


${ }^{1}$ Lomonosov Moscow State University, Faculty of Biology, 1-12 Leninskie Gory, Moscow, 119991 Russia, e-mail dariatodor@mail.ru ( $\square$ corresponding author), slatolya@mail.ru, matorin@biophys.msu.ru, gmaksimov@mail.ru; ${ }^{2}$ Shenzhen MSU-BIT University, No 299, Ruyi Road, Longgang District, Shenzhen, Guangdong, 518172 China, e-mail haojr@szmsubit.edu.cn;

${ }^{3}$ Maize Research Institute, Zemun Polje, ul. Slobodana Bajicha 1, 11185 Belgrade-Zemun, Serbia, e-mail radenovic@sbb.rs;

${ }^{4}$ National Research Technological University MISIS, 4, Leninskii prosp., Moscow, 119049 Russia, e-mail gmaksimov@mail.ru

ORCID:

Todorenko D.A. orcid.org/0000-0002-7344-0256

Hao J. orcid.org/0000-0003-4256-5969

Radenovich C.N. orcid.org/0000-0001-7218-9015

Matorin D.N. orcid.org/0000-0002-6164-5625

Slatinskaya O.V. orcid.org/0000-0002-9908-2637

Seifullina N.Kh. orcid.org/0000-0002-6313-794X

Maksimov G.V. orcid.org/0000-0002-7377-0773

The authors declare no conflict of interests

Acknowledgements:

Supported financially by Russian Foundation for Basic Research, grant No. 20-04-00465 A

Received July 30, 2019 doi: 10.15389/agrobiology.2020.1.66eng

\section{Abstract}

In the last decade, neonicotinoid insecticides have been actively used to protect plants from pests. Moreover, their effect on the plants, in particular on the state of photosynthetic pigments, has been studied paucity. In the present work, it was shown for the first time that treatment of maize (Zea mays L.) leaves with a thiamethoxam (TMX) insecticide leads to a decrease in the functional activity of photosystem II and a decrease in the energyzation of thylakoid membranes. In addition, the effect of thiamethoxam depends on the genotype of maize. The aim of the work was to study the effect of thiamethoxam pesticide on photosynthetic pigments and the photochemical activity of the photosynthetic apparatus of corn leaves of two genotypes. The experiments were carried out in 2018-2019. The object of the study was samples of maize leaves of the inbred line zppl 225 and hybrid zp 341 with high rates of germination, grain quality and yield (Institut za kukuruz "Zemun Polje", Belgrade, Serbia). The seeds were germinated until the roots appeared (length not less than $5 \mathrm{~mm}$ ), after which they were planted in the soil (vermiculite: chernozem mixture, 1:1) and grown under 16-hour daylight at a constant temperature of $25^{\circ} \mathrm{C}$. When the third true leaf appeared (more than $4 \mathrm{~cm}$ in length), the plants were sprayed with a TMX solution at a concentration of $0.2 \mathrm{mg} / \mathrm{l}$. Plants grown under similar conditions without TMX spraying were used as controls. The measurements were carried out when the fifth true leaf reached a size of $12-14 \mathrm{~cm}$. The content of photosynthetic pigments (chlorophyll a, b and carotenoids) was determined spectrophotometrically in $100 \%$ acetone extract and calculated using the Holm-Wetstein formula. A change in the conformation of carotenoid molecules was recorded by Raman spectroscopy. Light-induced kinetics of prompt fluorescence (PF), delayed fluorescence (DF) and modulated reflection at $\lambda=820 \mathrm{~nm}$ (MR) were recorded simultaneously using a multifunctional plant efficiency analyzer M-PEA-2 (Hansatech Instruments, Great Britain). PF induction curves (OJIP curves) were analyzed using a standard JIPtest. It was found that in the phase of the fifth true leaf, in the inbred line zppl 225, the chlorophyll content in the presence of TMX decreased from 0.74 to $0.61 \mathrm{mg} / \mathrm{g}$ : the amount of chlorophyll a decreased by $17 \%$, chlorophyll b by $24 \%$. In contrast, no changes in pigment composition were detected in the leaves of the zp 341 hybrid when exposed to TMX. The OJIP curves of the control and TMX-treated leaves had a typical curve with characteristic O-J, J-I, and I-P phases, which reflected the processes of sequential reduction of carriers in the electron transport chain of photosynthesis (ETC) between two photosystems. The effect of the pesticide on the leaves of two maize genotypes was manifested in a decrease in the functional state of photosystem II, determined by the fluorescence parameter $\left(\mathrm{PI}_{\mathrm{ABS}}\right)$, which was obtained based on the analysis of OJIP curves using the JIPtest. Comparison of $\mathrm{PI}_{\mathrm{ABS}}$ in the control and under the influence of TMX revealed statistically significant $(\mathrm{p}<0.05)$ differences: in the leaves of zppl 225 and zp 341 samples treated with TMX, the $\mathrm{PI}_{\mathrm{ABS}}$ parameter decreased by 29 and $24 \%$, respectively. Changes in the fast phase of delayed fluorescence, associated with a decrease in the energyzation of the thylakoid membrane upon exposure to TMX, were detected in the leaves of maize. An analysis of the maximum oxidation and reduction rates of P700 (MR kinetics) indicates a decrease in the acceptor pool on the acceptor side of PSI in zppl 225 leaves when exposed to TMX. It was found that the reaction centers (RCs) of PSI zp 341 showed resistance to TMX (no change in the redox transformations of P700). TMX caused changes in the conformation of carotenoid molecules, but did not change their content in the leaf. The proposed combination of methods for prompt fluorescence, delayed fluorescence, modulated reflection at $\lambda=820 \mathrm{~nm}$ and Raman spectroscopy can be the basis for the formation of an effective technology for the diagnosis of early defects of photosynthetic pigments when pesticides enter an intact plant.

Keywords: Zea mays L., pesticides, Raman spectroscopy, thiamethoxam, chlorophyll, carotenoids, chlorophyll fluorescence. 